\title{
Nor-isoprenoids profile during port wine ageing-influence of some technological parameters
}

\author{
António César Silva Ferreira, Paula Guedes de Pinho* \\ Escola Superior de Biotecnologia, Universidade Católica Portuguesa, Rua Dr. Bernardino de Almeida, 4200-072 Porto, Portugal
}

Keywords: Port wines; Ageing; Volatile nor-isoprenoid compounds; $\beta$-Damascenone; $\beta$-Ionone; 2,2,6-Trimethylcyclohexanone;

1,1,6-Trimethyl-1,2-dihydronaphthalene; Vitispirane

\begin{abstract}
Nor-isoprenoid compounds, such as $\beta$-damascenone, $\beta$-ionone, 2,2,6-trimethylcyclohexanone (TCH), 1,1,6-trimethyl-1,2-dihydronaphthalene (TDN) and vitispirane were determined in 14 young port wines and 45 old port wines. As between the two groups of wines levels of these compounds are quite different, an experimental protocol was performed in order to determine which technological parameter (dissolved $\mathrm{O}_{2}$, free $\mathrm{SO}_{2}$ levels, $\mathrm{pH}$ and time/temperature) was related with the formation/consumption of these molecules. The five nor-isoprenoids were equally affected by the selected parameters and a similar profile with time was observed. The synergistic effects of increasing temperature and lowering $\mathrm{pH}$ had the largest impact. For samples treated with high oxygen regimes (saturation), the levels of all considered nor-isoprenoids decreased after a certain concentration of oxygen consumed (e.g. $10 \mathrm{mg} \mathrm{l}^{-1}$ ).

Nevertheless, during barrel port wine ageing, corresponding to the 45 wines, two different behaviours can be observed: TDN, vitispirane and TCH increase significantly whilst a decrease of levels of $\beta$-ionone and $\beta$-damascenone with port barrel ageing was observed. It was also calculated that "over 40 year" old port wines have, respectively, 15, 5 and 3 times higher levels of TDN, vitispirane and TCH than the young ports. For these three compounds the respective rates of formation are higher than those of degradation, which suggests a higher number or higher concentration of precursors than those involved for the megastigame $\mathrm{C} 13$ nor-isoprenoids $\beta$-damascenone and $\beta$-ionone.
\end{abstract}

\section{Introduction}

Nor-isoprenoids are substances that could come from the direct degradation of carotenoid molecules such as $\beta$ carotene, lutein, neoxanthin and violaxanthin [1-3] and also from the hydrolysis of glucoside molecules [4-6]. Some nor-isoprenoids have been identified in wine, such as $\beta$-damascenone, $\beta$-ionone, 1,1,6-trimethyl-1,2-dihydronaphthalene (TDN) and vitispirane. All these molecules have an important sensorial impact on wine aroma as they have very low olfactory perception thresholds. Some studies have been published concerning the volatile composition of port wine [2,3,5-8]. Although, there is little information concerning the levels of nor-isoprenoids in ports, recently some authors [4] have reported the presence of 2,2,6-trimethylcyclohexanone (TCH), responsible for the "rock-rose-like" aroma in port wines.

* Corresponding author. Fax: +351-2-5580088.

E-mail address: pinho@esb.ucp.pt (P. Guedes de Pinho).
It is interesting to note that port wines, especially for bottle aged ("vintage category") develop "floral" and "violet" notes, which could be related to the presence of some of these specific molecules. As port wines have a short period of fermentation, as it is interrupted by brandy addition, some constituents in grape material can be found in the respective wines. This is the case of carotenoid molecules such as $\beta$ carotene, lutein, neoxanthin and violaxanthin, which were reported for the first time in young port wines [7]. Thus, considering on one hand the presence of free carotenoid molecules in port wines and for another hand, the long ageing period that ports could be submitted, with or without oxygen presence (barrel aged ports or "vintage" ports, respectively), it seems to be interesting to determine, firstly the contents of nor-isoprenoids in different categories of ports and also their evolution during ageing.

Hence, the aim of this work was to relate changes in the composition of nor-isoprenoids during port wine ageing and to determine among technological parameters such as dissolved $\mathrm{O}_{2}, \mathrm{pH}$ and temperature which 
have the highest impact on formation/degradation of these compounds.

For this reason an experimental protocol was established in order to analyse the effect of some parameters which are believed to be important in the aromatic modification of port wines, namely temperature, oxygen concentration, $\mathrm{pH}$ and $\mathrm{SO}_{2}$ concentration.

\section{Materials andmethods}

\section{Material}

The wines used in this study were: Wine Group 1-young port wines, from one particular vintage (1999), from five different cultivars of Vitis vinifera varieties (Touriga Nacional, Touriga Francesa, Tinta Roriz, Tinto Cão and Tinta Barroca) from two different sub regions of the Douro Region (Cima Corgo and Douro Superior). Winemaking procedures depended on the producers. Wine Group 2-10, 20, 30 and over 40-year-old tawny port. These wines were blended and barrel aged. Wine Group 3-red port wine from 1999 vintage (81), with $\mathrm{pH} 3.74$, an amount of dissolved oxygen of $4.2 \mathrm{ml}$, with an instantaneous potential of $222 \mathrm{mV}$ and a free $\mathrm{SO}_{2}$ level of $17 \mathrm{mgl}^{-1}$, was prepared in order to analyse the effect of parameters which are believed to be important in the aromatic modification of port wines, namely: temperature, oxygen concentration, $\mathrm{pH}$ and $\mathrm{SO}_{2}$ concentration (Fig. 1). To test the effect of $\mathrm{pH}$, one of the portions was adjusted to $\mathrm{pH} 3.0$ by adding tartaric acid. To test the effect of high concentrations of oxygen (oxidative environment) and the effect of an exogenous antioxidant, a portion of wine at $\mathrm{pH}$ 3.0 was, saturated with oxygen $\left(6.2 \mathrm{mg} \mathrm{l}^{-1}\right)$ by air bubbling, and finally to the last portion of wine at $\mathrm{pH} 3.0$, free $\mathrm{SO}_{2}$ was adjusted to $74 \mathrm{mg} \mathrm{l}^{-1}$ by adding potassium metabisulphite.

As shown in Fig. 1, the four portions, each 1.51, were divided into sets of $100 \mathrm{ml}$ and were stored in sealed vessels. One part of each experiment was kept at $15^{\circ} \mathrm{C}$, another at $45^{\circ} \mathrm{C}$ and the last one at $60^{\circ} \mathrm{C}$. The samples were analysed at $0,17,32,47$ and 59 days of storage time. The samples initially saturated with oxygen were re-saturated at each sampling stage. This experiment was performed in duplicate.

\section{Analyticaldeterminations}

To $50 \mathrm{ml}$ of white wine were added $50 \mu \mathrm{l}$ of octan-3-ol in hydroalcoholic solution $(1 / 1 \mathrm{v} / \mathrm{v})$ at $466 \mathrm{mg} \mathrm{l}^{-1}$ as internal standard and $5 \mathrm{~g}$ of anhydrous sodium sulphate. The wine was extracted twice with $5 \mathrm{ml}$ of $\mathrm{CH}_{2} \mathrm{Cl}_{2}$ (Merck). The two organic phases obtained were blended and dried over anhydrous sodium sulphate. Four millilitres of this organic extract was concentrated until $1 / 10$ under a nitrogen stream with a $201 \mathrm{~min}^{-1}$ gas flow [8].

\section{Ga schromatography/mass spectrometry (GC/MS)}

Extracts were analysed using a Varian CP-3800 gas chromatograph equipped with a Varian Saturn 2000 mass selective detector and Saturn GC/MS workstation software version 5.51. The column used was STABILWAX-DA $(60 \mathrm{~m} \times 0.25 \mathrm{~mm}, 0.25 \mu \mathrm{m})$ fused silica (Restek). The injector port was heated to $220^{\circ} \mathrm{C}$. The split vent was opened after $30 \mathrm{~s}$. The carrier gas was helium C-60 (Gasin, Portugal), at $1 \mathrm{ml} \mathrm{min}^{-1}$, constant flow. The oven temperature was $40{ }^{\circ} \mathrm{C}$ (for $1 \mathrm{~min}$ ), then increased at $2{ }^{\circ} \mathrm{C} \mathrm{min}^{-1}$ to $220^{\circ} \mathrm{C}$ and held for $30 \mathrm{~min}$. All mass spectra were acquired in the electron impact (EI) mode. The ion trap detector was set as follows: the transfer line, manifold and trap temperatures were, respectively, 230,45 and $170^{\circ} \mathrm{C}$. The mass range was $m / z 33-350$, with a scan rate of $6 \mathrm{~s}^{-1}$. The emission current was $50 \mu \mathrm{A}$, and the electron multiplier was set in relative mode to the autotune procedure. The maximum ionisation time was $25,000 \mu \mathrm{s}$, with an ionisation storage level of $\mathrm{m} / \mathrm{z}$ 35. The injection volume was $1 \mu \mathrm{l}$ and the analysis was performed in full scan mode. Identification was achieved from comparisons of mass spectra obtained from the sample with those from pure standards injected in the same conditions; by comparing the Kovats indices and the mass spectra present in the NIST 98 MS Library Database or in

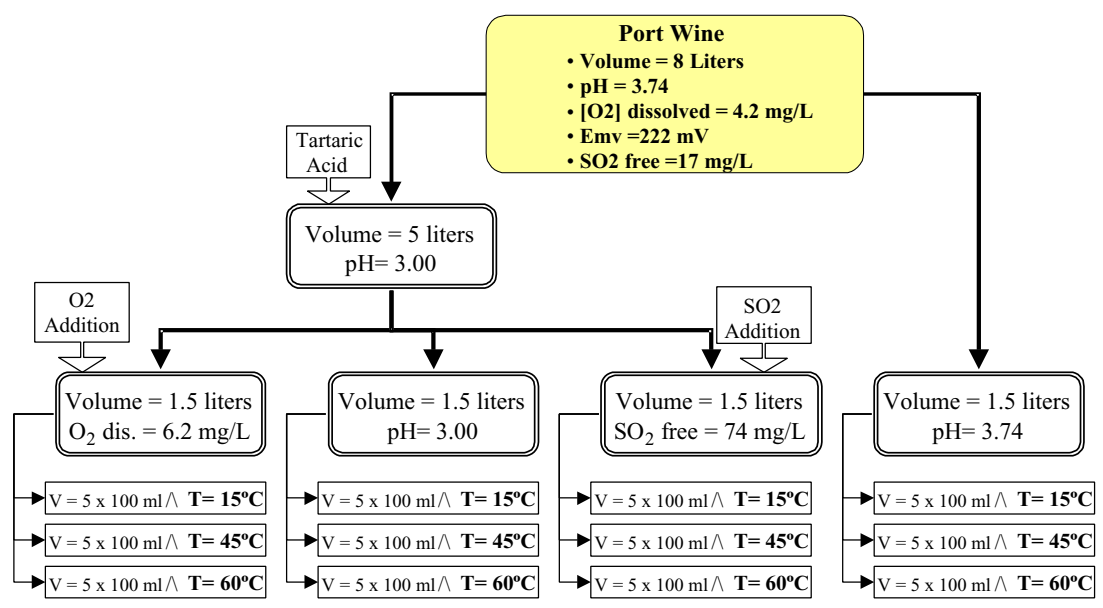

Fig. 1. Schematic representation of the preparation of the port wine samples (Wine Group 3). 
the literature. The Kovats index is calculated according to the literature [9].

\section{Other analytical measurements}

Measurements of redox potential, free $\mathrm{SO}_{2}$ concentration, and chromatic index were made [10]. The concentration of dissolved oxygen was measured using a "WTW 340 Oxygen Probe".

\section{Standards}

The following molecules were purchased from SigmaAldrich: 3-octanol (20296-29-1) (99\%), $\beta$-ionone (1490107-6) (96\%) and 2,2,6-trimethylcyclohexanone (T7.573-6) $(98 \%)$. The $\beta$-damascenone was kindly supplied by Firmenich (Switzerland). 1,1,6-Trimethyl-1,2-dihydronaphthalene was synthesised according to [11]. The degree of purity obtained was $<30 \%$, due to the presence of $1,1,6-$ trimethyl-1,2,3,4-tetrahydronaphthalene (TTN) sufficient to use for GC/MS identification. Vitispirane was identified by Kovats index and mass spectrum according to the literature [9].

\section{Statistical methods}

An analysis of variance (ANOVA) using Excel ${ }^{\mathrm{TM}}$ software from windows 95 v. 7.0. was applied to the experimental data; the results were considered significant if the associated $P$-value was $<0.05$.

\section{Results and discussion}

The five compounds: $\beta$-ionone, $\beta$-damascenone, 2,2,6trimethylcyclohexanone, vitispirane and 1,1,6-trimethyl-1,2naphthalene, were selected as they were the major nor-isoprenoids found in wines [12-16].

The quantification of these compounds in young and aged port wines show two different evolution trends with time of storage. The levels on $\mathrm{C}$-13 nor-isoprenoids $\beta$-ionone and $\beta$-damascenone decreases and the concentration of $\mathrm{TCH}$, vitispirane and TDN increases with ageing (Table 1).

It must be stressed that vintage port wines (bottle aged wines), develop "floral" and "violet" notes which could be related to the presence of C13 nor-isoprenoids. Conversely for old barrel aged port of equivalent age these descriptors are not found. This fact could be related to the different oxygen contents.

In order to gather more information concerning the former behaviour, an experimental protocol (Fig. 1) was implemented as described in Section 2.

\section{1,1,6-Trimethyl-1,2-dihydronaphthalene}

It has already been shown that TDN is formed during bottle storage [12]. It has also been demonstrated that
TDN is a very important key odourant related to the typical aroma of oxidation-spoiled white wines [17,18]. Some works have shown that TDN is a direct degradation product of $\beta$-carotene [19]. Other authors [20] propose another precursor involving megastigme-4,7-dien-3,6,9-triol linked to a sugar molecule. Finally, Winterhalter [21] proposes another precursor which exists in grapes linked to a sugar, the 2,6,10,10-tetramethyl-1-oxaspiro[4,5]dec-6-ene-2,8-diol.

According to this author TDN is not present in grapes, but is formed during ageing, and levels near $200 \mu \mathrm{gl}^{-1}$ can be found in some old aged Riesling wines [21]. Results obtained from the protocol experiment showed that $\mathrm{pH}$ and temperature are the most important parameters in the formation of TDN (Fig. 2). The behaviour of TDN related to oxygen is quite interesting. These results corroborate some others obtained for a white wine degradation [17]. Nevertheless, old port wines, aged over 40 years in barrels, have levels of TDN 15 times higher than young port wines and 4.6 times than that of 10-year-old port wines (Table 1). It seems that oxygen promotes its formation, and higher levels of $\mathrm{O}_{2}$ increases its rate of degradation. In barrel aged wines, the number and/or the quantities of precursors have much greater influence on the formation of TDN than the presence of oxygen.

Wines with low $\mathrm{pH}$ are more sensitive to the formation of TDN (Fig. 2). In fact, Riesling wines coming from cold regions with low $\mathrm{pH}$, have higher levels of TDN than the same wines produced on the south of Europe, for example [14], which confirm the presence of a TDN precursor linked to a sugar molecule which is broken down preferentially at low $\mathrm{pH}$.

\section{Vitispirane}

This compound was identified in wine by Simpson et al. [16]. TDN vitispirane has as precursor a megastigme compound (megastigme-4,7-dien-3,6,9-triol), which is linked to a sugar molecule. The olfaction descriptor of vitispirane is "camphor" or "eucalyptus" [22]. Some previous studies show that for ruby port wines the presence of vitispirane can attain the perception threshold $\left(800 \mu \mathrm{gl}^{-1}\right)$, consequently it participates in wine aroma [23]. Old port wines, between 10 years old and over 40 years old, have two to five times higher vitispirane levels than young port wines. The variation in the vitispirane concentration during the experimental period is shown in Fig. 3. As mentioned for TDN, the $\mathrm{pH}$ and temperature are the two factors that have the greatest influence on its formation. During the first days of this study, the rate of formation was higher than later in the study, which can be confirmed from other authors' work [16]. Samples submitted to an oxygen treatment increased their levels during the first days of the experimental protocol, however after 24 days levels on vitispirane decreased; this same behaviour occurred for TDN. Extremely high levels of $\mathrm{O}_{2}$ saturation could dramatically increase vitispirane degradation. 
Table 1

Concentration $\left(\mu \mathrm{gl}^{-1}\right)$ of some nor-isoprenoid compounds in young and old port wines

\begin{tabular}{|c|c|c|c|c|c|}
\hline Port wine & $\beta$-Damascenone & $\beta$-Ionone & $\mathrm{TCH}$ & Vitispirane $^{\mathrm{a}}$ & $\mathrm{TDN}^{\mathrm{a}}$ \\
\hline \multicolumn{6}{|l|}{ Young $^{b}$} \\
\hline Max & 13.4 & 1.4 & 0.8 & 0.8 & 1.9 \\
\hline Third quartile & 11.3 & 0.9 & 0.7 & 0.5 & 0.5 \\
\hline Median of 14 wines & 7.4 & 0.6 & 0.5 & 0.4 & 0.3 \\
\hline First quartile & 5.6 & 0.5 & 0.3 & 0.3 & 0.2 \\
\hline Min & 4.5 & 0.3 & 0.2 & 0.2 & 0.1 \\
\hline \multicolumn{6}{|l|}{ 10-year-old } \\
\hline $\operatorname{Max}$ & 3.9 & 0.3 & 1.1 & 1.2 & 2.3 \\
\hline Third quartile & 3.5 & 0.3 & 1.0 & 1.0 & 1.5 \\
\hline Median of 12 wines & 3.1 & 0.2 & 0.9 & 0.8 & 0.9 \\
\hline First quartile & 2.6 & 0.2 & 0.9 & 0.6 & 0.5 \\
\hline Min & 1.3 & nd & 0.8 & 0.5 & 0.3 \\
\hline \multicolumn{6}{|l|}{ 20-year-old } \\
\hline Max & 5.1 & 0.4 & 1.6 & 1.7 & 3.9 \\
\hline Third quartile & 4.3 & 0.3 & 1.3 & 1.7 & 2.9 \\
\hline Median of 10 wines & 3.7 & 0.2 & 1.2 & 1.2 & 2.3 \\
\hline First quartile & 2.6 & 0.1 & 1.1 & 1.0 & 1.9 \\
\hline Min & 1.8 & nd & 0.7 & 0.8 & 1.3 \\
\hline \multicolumn{6}{|l|}{ 30-year-old } \\
\hline Max & 4.8 & 0.4 & 2.2 & 3.0 & 5.2 \\
\hline Third quartile & 3.9 & 0.3 & 1.6 & 1.7 & 3.5 \\
\hline Median of 12 wines & 2.9 & 0.3 & 1.5 & 1.3 & 2.9 \\
\hline First quartile & 2.5 & 0.2 & 1.2 & 1.0 & 2.0 \\
\hline Min & 1.9 & nd & 1.0 & 0.7 & 1.3 \\
\hline \multicolumn{6}{|l|}{ Over 40 years old } \\
\hline Max & 5.0 & 0.7 & 2.7 & 2.3 & 7.7 \\
\hline Third quartile & 4.3 & 0.4 & 2.0 & 2.3 & 5.3 \\
\hline Median of 9 wines & 3.8 & 0.4 & 1.4 & 1.9 & 4.9 \\
\hline First quartile & 3.3 & 0.3 & 1.2 & 1.5 & 3.7 \\
\hline Min & 3.0 & nd & 1.1 & 1.4 & 1.5 \\
\hline
\end{tabular}

Max: maximum value, Min: minimum value, TCH: trimethylcyclohexanone, TDN: 1,1,6-trimethylnaphthalene, nd: not detected.

a Compound area/internal standard area.

b Wines from 1999 vintage, five different cultivars, two different subregions, with different winemaking procedures.

The relationship between TDN and vitispirane is not linear but logarithmic (Fig. 4). This observation suggested similar formation conditions but arising from different precursors as reported [20-23]. TDN is formed simultaneously with vitispirane, the relative rate of formation of TDN being higher than that of vitispirane.

\section{2,2,6-Trimethylcyclohexanone}

The nor-isoprenoids with no-megastigme structure such as the dihydroactinolide (C11) and the 2,2,6-trimethylcyclohexanone (C9) can be formed by thermal degradation of $\beta$-carotene [2]. Temperature is the major factor that affects

\section{1,1,6-Trimethyl-1,2-dihydronaphthalene}

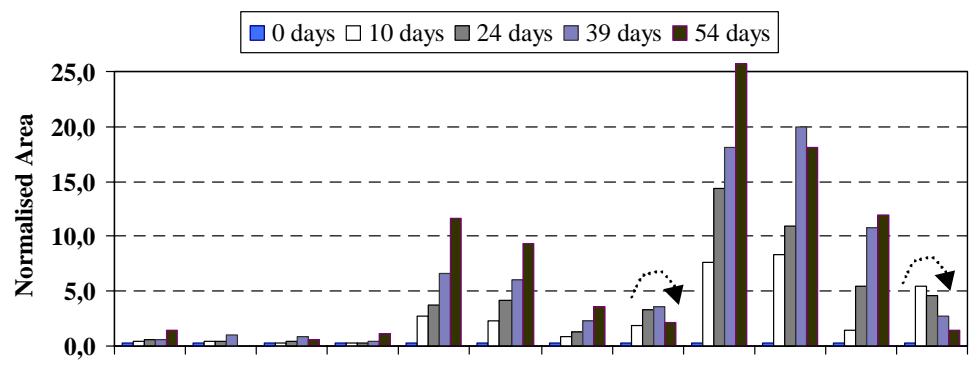

pH3; SO2; pH4; Ox; pH3; SO2; pH4; Ox; pH3; SO2; pH4; Ox; $15{ }^{\circ} \mathrm{C} 15^{\circ} \mathrm{C} 15^{\circ} \mathrm{C} 15^{\circ} \mathrm{C} 45^{\circ} \mathrm{C} 45^{\circ} \mathrm{C} 45^{\circ} \mathrm{C} 45^{\circ} \mathrm{C} 60^{\circ} \mathrm{C} 60^{\circ} \mathrm{C} 60^{\circ} \mathrm{C} 60^{\circ} \mathrm{C}$

Treatments

Fig. 2. 1,1,6-Trimethyl-1,2-dihydronaphthalene concentration in samples after 54 days storage. 


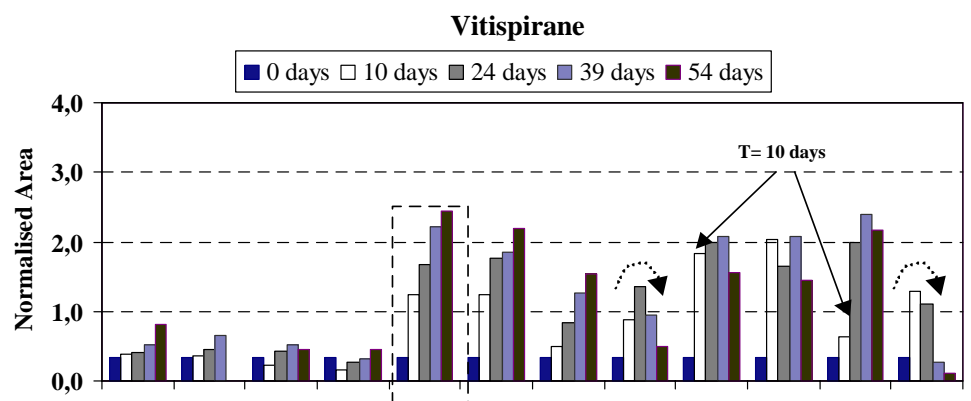

pH3; SO2; pH4; Ox; 1 pH3; 'SO2; pH4; Ox; pH3; SO2; pH4; Ox; $15^{\circ} \mathrm{C} 15^{\circ} \mathrm{C} 15^{\circ} \mathrm{C} \quad 15^{\circ} \mathrm{C}, 45^{\circ} \mathrm{C}, 45^{\circ} \mathrm{C} 45^{\circ} \mathrm{C} \quad 45^{\circ} \mathrm{C} \quad 60^{\circ} \mathrm{C} \quad 60^{\circ} \mathrm{C} \quad 60^{\circ} \mathrm{C} 60^{\circ} \mathrm{C}$

Treatments

Fig. 3. Vitispirane concentration in samples after 54 days storage.

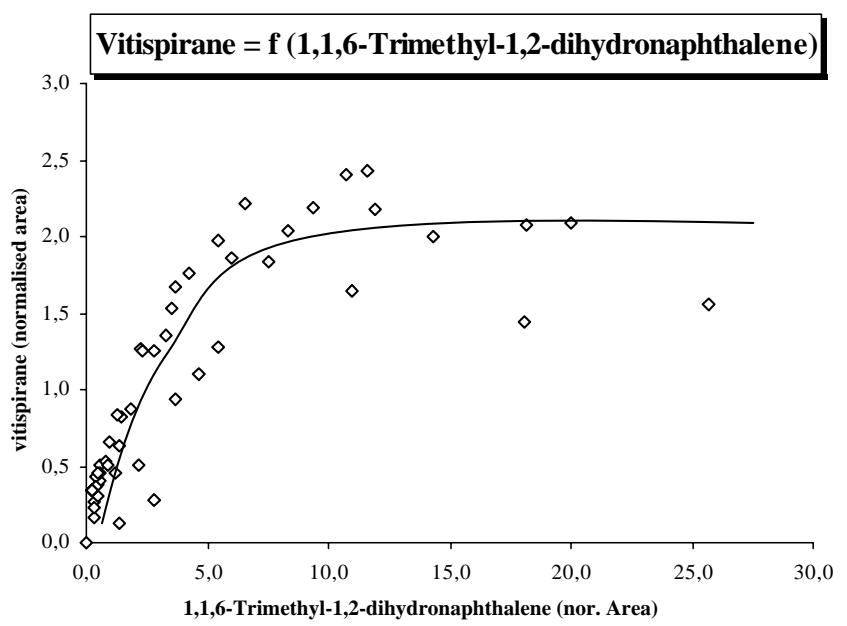

Fig. 4. Relationship between vitispirane and TDN, concentrations.

the increases in levels of this substance. TCH was identified in tea and in wine samples [15,24], on wine it participates on the "rock-rose-like" aroma. As occurs with other nor-isoprenoids no-megastigme such as TDN the acidic $\mathrm{pH}$ favours its formation. The ANOVA procedure showed significant differences for TCH concentrations due to $\mathrm{pH}$ treat- ment. Among the four treatments for samples stored at $45^{\circ} \mathrm{C}$, the $P$-value $=0.0230$ at the $95 \%$ level, for samples stored at $60{ }^{\circ} \mathrm{C}$, the $P$-value $=0.044$ at the $95 \%$ level. At those temperature the amount of this compound in the samples at low $\mathrm{pH}$ increases clearly with time The saturation of samples with oxygen does not have an important impact (Fig. 5). In fact in old port wines levels can reach $2.7 \mu \mathrm{gl}^{-1}$, compared with an average of $0.5 \mu \mathrm{g} \mathrm{l}^{-1}$ in young port wines (Fig. 6).

\section{(2,6,6-Trimethyl-1-cyclohexen-1-yl)-3-buten-2-one ( $\beta$-ionone)}

This ketone has a very low perception threshold, $4.5 \mu \mathrm{gl}^{-1}$ [25] and can participate in the wine aroma. Its descriptor is "violet". $\beta$-Ionone can be formed directly by $\beta$-carotene degradation [2] or by its sugar precursor hydrolysis [13]. Young port wines have higher contents on $\beta$-ionone than old barrel aged port wines. As shown in Fig. 7, all treatments at $15^{\circ} \mathrm{C}$ had no impact on the $\beta$-ionone concentration. The ANOVA procedure showed no significant differences for $\beta$-ionone concentrations among the four treatments for samples stored at $15^{\circ} \mathrm{C}$. However, treatments at 45 and $60^{\circ} \mathrm{C}$ led to increases in its levels. Low $\mathrm{pH}$ treatments had a great impact on its concentration,

2,2,6-trimethyl-2-cyclohexanone

$\square 0$ days $\square 10$ days $\square 24$ days $\square 39$ days $\square 54$ days

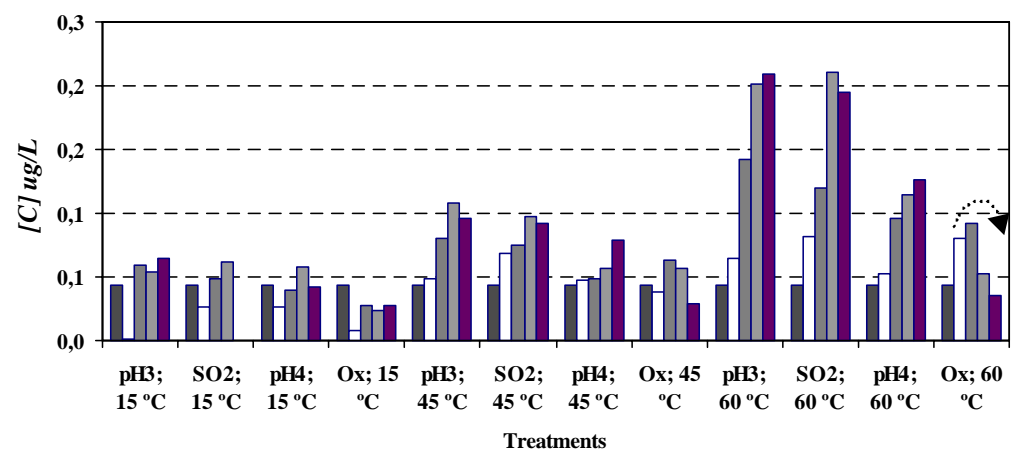

Fig. 5. 2,2,6-Trimethyl-2-cyclohexanone concentration in samples after 54 days storage. 


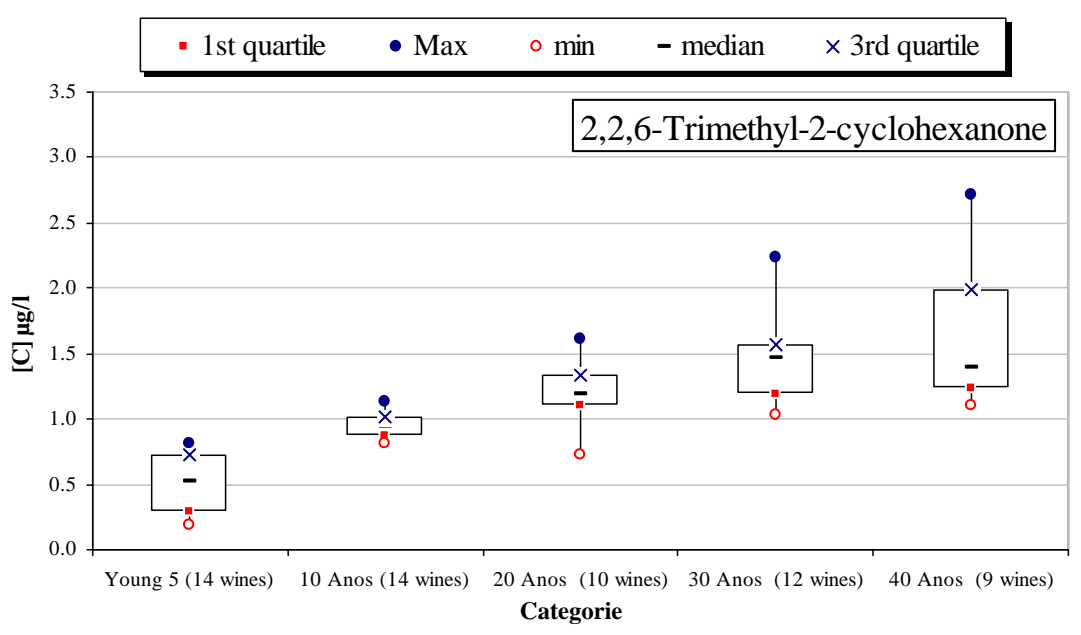

Fig. 6. Box plot representing the variation of $\mathrm{TCH}$ concentration in the analysed port wines.

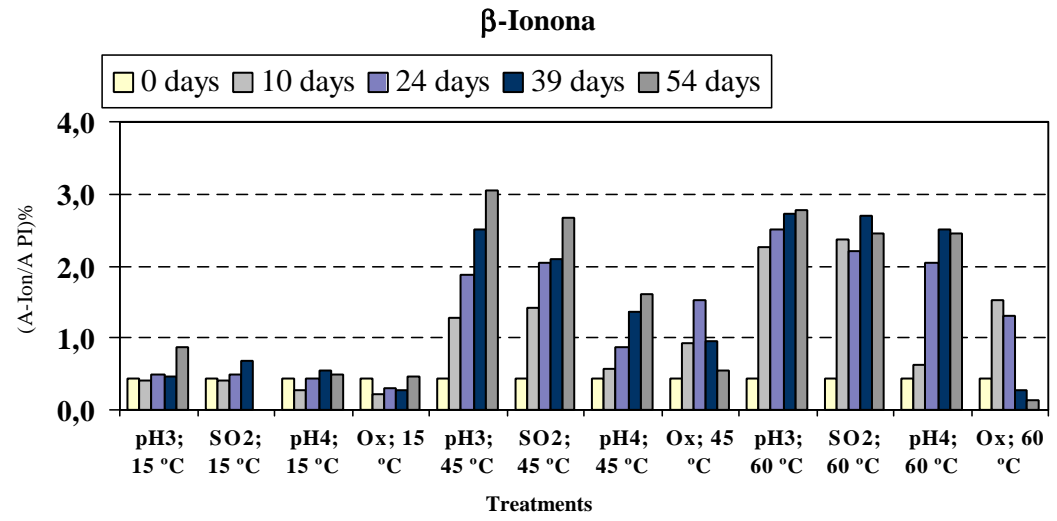

Fig. 7. $\beta$-Ionone concentration in samples after 54 days of storage.

as well as higher temperatures which increase the rate of formation of $\beta$-ionone, as can be seen if, the results at $\mathrm{pH}$ 3 at 45 and $60^{\circ} \mathrm{C}$ are compared. Finally, levels of $\beta$-ionone decreased with oxygen treatment after 39 days. Oxygen can be considered an important factor in the $\beta$-ionone degradation. Conversely, the presence of $\mathrm{SO}_{2}$ retards its degradation. Kanasawud and Crouzet [2] report that $\beta$-ionone can lead to the formation of 2,2,6-trimethylcyclohexanone. However, according to results presented in Fig. 8, there is a concomitant formation of these two molecules with a high dependence between them.

\section{(2,6,6-Trimethyl-1,3-cyclohexadien-1-yl)-2-buten-1-one} ( $\beta$-damascenone)

Among nor-isoprenoids, $\beta$-damascenone is one of the most important substances with a low perception olfaction threshold. The origin of this compound on wine is not well established; there are two main pathways possible, via acid hydrolysis [3,6] and direct degradation of neoxanthin [26]. Results shown in Fig. 9 confirm these two possible origins of $\beta$-damascenone. Higher levels were found for $\mathrm{pH} 3.0$ treatment and for temperatures 45 and $60^{\circ} \mathrm{C}$. At these temperatures the amount of this compound in the samples at $\mathrm{pH} 3$ increases clearly with time. The low $\mathrm{pH}$ favours the acid hydrolysis, whereas higher tem-

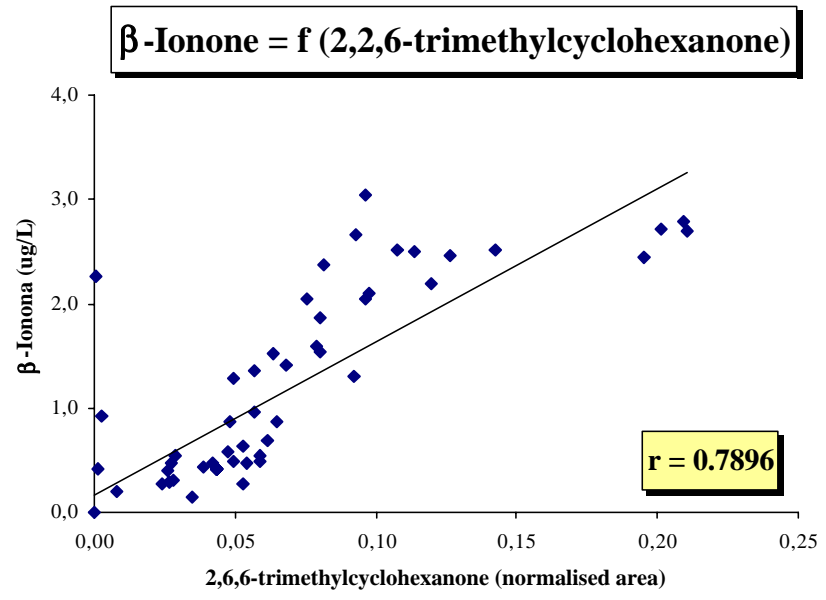

Fig. 8. Relationship between $\beta$-ionone and 2,2,6-trimethylcyclohexanone concentrations. 


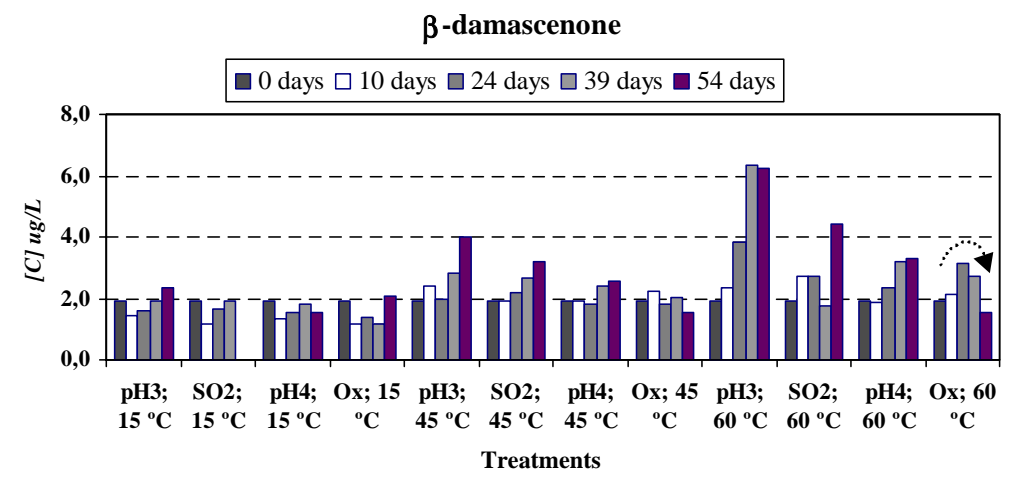

Fig. 9. $\beta$-Damascenone concentration in samples after 54 days storage.

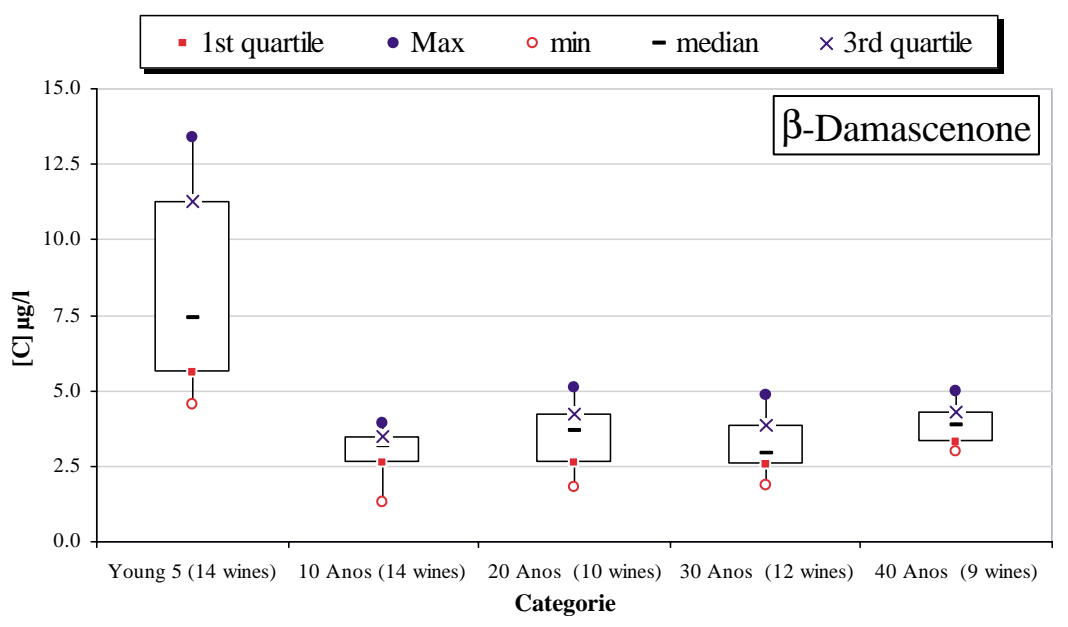

Fig. 10. Box plot representing the variation of $\beta$-damascenone concentration in the analysed port wines.

peratures favour the direct degradation of neoxanthin. As occurs for other nor-isoprenoids $\beta$-damascenone increased in samples saturated with oxygen for the first days of the study, after which there was a decrease. $\mathrm{SO}_{2}$ affects the rate of formation of $\beta$-damascenone. This can be observed by comparing the sample at $\mathrm{pH} 3.0$ with $\mathrm{SO}_{2}$ at 45 and $60^{\circ} \mathrm{C}$. The comparison between young and old port wines (Fig. 10) show that levels of this compound decreased with ageing.

\section{Conclusions}

Some old port wines develop aromas which can be related to the presence of some nor-isoprenoids, such as $\beta$-ionone and $\beta$-damascenone for old vintage wines, and vitispirane, 2,2,6-trimethylcyclohexanone and TDN for old barrel aged wines. Old port wines, aged over 40 years in barrels have levels of TDN 15 times higher than young port wines and 4.6 times higher than 10-year-old port wines.

It was demonstrated that TDN, vitispirane and $\mathrm{TCH}$ present in very low levels in the initial wine are formed during the experimental period, preferentially at low $\mathrm{pH}$ values and high temperatures $\left(\geq 45^{\circ} \mathrm{C}\right)$. Samples submitted to oxygen treatment had an increase in vitispirane concentration during the beginning of the study, however, after 24 days the levels decreased; this same behaviour occurs for TDN. Extremely high levels of $\mathrm{O}_{2}$ saturation can drastically promote vitispirane and TDN degradation.

The presence of free sulphur dioxide decreased levels of TDN formed and avoided the oxidative degradation of vitispirane. High concentrations of dissolved oxygen $\left(6 \mathrm{mg} \mathrm{l}^{-1}\right)$, which signify very drastic wine oxidation, had little influence on the formation of these molecules but promoted its degradation. It was verified that levels of TDN and vitispirane have a logarithmic dependence, which could predict the existence of different precursors.

The decreasing of $\beta$-ionone concentration is dependent on the oxygen content. The use of $\mathrm{SO}_{2}$ and the absence of $\mathrm{O}_{2}$ can avoid its degradation during ageing.

As occurs for other nor-isoprenoids the damascenone concentration increased in samples saturated with oxygen during first days of the study, after which there is a decrease. $\mathrm{SO}_{2}$ affects the rate of formation of $\beta$-damascenone. The comparison between young and old port wines shows that this compound decreased with barrel ageing. 


\section{Acknowledgements}

The work was suported by a MCT/FCT/POCTI/AGR/ $42598 / 2001$ project.

\section{References}

[1] C.R. Mordi, J.C. Walton, G.W. Burton, L. Hughes, K.U. Ingold, D.A. Lindsay, Exploratory study of $\beta$-carotene auto oxidation, Tetrahedron Lett. 32-33 (1991) 4203.

[2] P. Kanasawud, J.C. Crouzet, Mechanism of formation of volatile compounds by thermal degradation of carotenoids in aqueous medium, J. Agric. Food Chem. 38 (1990) 237.

[3] P. Winterhalter, in: R. Teranishi, G. Takeoka, M. Guntert (Eds.), Flavour Precursors-Thermal and Enzymatic Conversion, ACS series, vol. 490, 1993, p. 98

[4] Y.Z. Gunata, C.L. Bayonove, R.L. Baumes, R.E. Cordonnier, The aroma of grapes. I. Extraction and determination of free and glycosodically bound fractions of some grape aroma components, J. Chromatogr. 331 (1985) 83.

[5] R. Stephano, S. Bottero, R. Pigello, D. Borsa, G. Bazzo, L. Corno, Glycosylated aroma precursors in red winemaking grape cv., Enotecnico 34 (1988) 63.

[6] G.K. Skouroumounis, R.A. Massy-Westropp, M.A. Sefton, P.J. Williams, Precursors of damascenone in fruit juices, Tetrahedron Lett. 33 (1992) 3533.

[7] P. Guedes de Pinho, A.C. Ferreira, J. Gomez Benitez, M. Mendes Pinto, T. Hogg, Determination of carotenoid profiles in grapes, J. Agric. Food Chem. 49 (2001) 5484

[8] A.C. Silva Ferreira, Caracterisation du Vieillissement du Vin de Porto. Approche Chimique et Statistique. Role Aromatique du Sotolon., These de Doctorat de L'Université Victor Segalen Bordeaux II, 1998, p. 593.

[9] H. Van Den Dool, P.D. Kratz, A generalization of the retention index system including linear temperature programmed gas-liquid partition chromatography, J. Chromatogr. 11 (1963) 463.

[10] J. Ribereau-Gayon, E. Peynaud, P. Ribereau-Gayon, P. Sudraud, Traité d'Oenologie, Sciences et Techniques du Vin, Tome 1, Analyse et contrôle des vins, Dunod, Paris, 1976.

[11] R. Schneider, A. Razungles, C. Augier, R. Baume, Monoterpenic and nor-isoprenoidic glycoconjugates of Vitis vinifera L., cv. Melon B. as precursors of odorants in Muscadet wines, J. Chromatogr. A 936 (2001) 145
[12] R.F. Simpson, (a) Aroma and compositional changes in wine with oxidation, storage and ageing, Vitis 17 (1978) 274.

[13] Y. Kotseridis, Eude de l'arome des vins de Merlot et CabernetSauvignon de la region Bordelaise, These de Doctorat de L'Université Victor Segalen Bordeaux II, 1999, p. 652.

[14] R.F. Simpson, Aroma composition of bottle aged wine, Vitis 18 , $148-154$

[15] V. Freitas, P. Ramalho, Z. Azevedo, A. Macedo, Identification of some volatile descriptors of the rock-rose like aroma of fortified red wines from Douro Demarcated Region, J. Agric. Food Chem. 47 (1999) 4327

[16] R.F. Simpson, C.R. Strauss, P.J. Williams, Vitispirane: a C13 spiroether in the aroma volatiles of grapes juice, wines and distilled grape spirits, Chem. Ind. 6 (1977) 633.

[17] A.C. Silva Ferreira, P. Guedes de Pinho, P. Rodrigues, T. Hogg, Kinetics of oxidative degradation of white wines and how they are effected by selected technological parameters, J. Agric. Food Chem. 50 (2002) 5919.

[18] A.C. Silva Ferreira, T. Hogg, P. Guedes de Pinho, Identification of key-odorants related to the typical aroma of oxidation-spoiled white wines, J. Agric. Food Chem. 51 (2003) 1373.

[19] K.E. Murray, J.E. Shipton, F.B. Whitfield, The chemistry of food flavour. I. Volatile constituents of passion fruit Passiflora edulis, Aust J. Chem. 25 (1972) 1921-1933.

[20] C.R. Strauss, E. Dimitriadis, B. Wilson, P.J. Williams, Studies on the hydrolysis of two megastigma-3,6,9-triols rationalizing the origins of some volatile $\mathrm{C} 13$ nor-isoprenoids of Vitis vinifera grapes, J. Agric. Food Chem. 34 (1986) 145.

[21] P. Winterhalter, 1,1,6-trimethyl-1,2-dihydronaftalene (TDN) formation in wine. 1. Studies on the hydrolysis of 2,6,10,10-tetramethyl1-oxaspiro[4.5]dec-6-ene-2,8-diol rationalising the origin of TDN and related C13 nor-isoprenoides in Riesling wine, J. Agric. Food Chem. 39 (1991) 1825.

[22] A. Rapp, H. Mandery, New progress in vine and wine research, Experientia 42 (1986) 873.

[23] A.A. Williams, M.J. Lewis, H.V. May, The volatile flavour components of commercial port wines, J. Sci. Food Agric. 343 (1983) 11

[24] M. Shimoda, H. Shigematsu, H. Shiratsuchi, Y. Osajima, Comparation of the odour concentrates by SDE and adsorptive column method from Green Tea infusion, J. Agric. Food Chem. 43 (1995) 1616.

[25] P.X. Etievant, Wine, in: H. Maarse (Ed.), Volatile Compounds in Food and Beverages, TNO-CIVO Food Analysis Institute, Zeist, The Netherlands, 1991, pp. 483-546.

[26] G.K. Skouroumounis, R.A. Massy-Estropp, M.A. Sefton, P.J. Williams, Synthesis of glucosides related to grape and wine aroma precursors, J. Agric. Food Chem. 43 (1995) 974 